\title{
„Da wird viel Schaden angerichtet“ - Grundschulkinder in der Pandemie
}

Die Probleme für Grundschulkinder und ihre Familien in der Pandemie sind vielfältig, die Folgen immens und die Langzeitfolgen noch gar nicht absehbar. In diesem Beitrag werden die Probleme und Folgen vorrangig aus dem Blickwinkel der Eltern dargestellt, denn beide Autorinnen haben Kinder an verschiedenen Grundschulen. Überdies engagieren sich beide als Gründerinnen einer Elterninitiative namens \#lautfürfamilien, die überwiegend in Nordrhein-Westfalen aktiv ist und kennen die Sorgen und Nöte vieler Eltern daher sehr gut. Aufgrund der beruflichen Expertise spielen bei dieser Analyse allerdings auch pädagogische und juristische Aspekte eine hervorgehobene Rolle.

\section{Probleme in der Pandemie - ein Überblick}

Zunächst geht es darum, die wichtigsten Probleme von Kindern, aber auch der Eltern zu identifizieren. Neben den offensichtlichen Bildungsverlusten spielen die psychischen und sozialen Folgen bei Kindern die größte Rolle. Dazu kommen Suchtverhalten, Gewalt und Kindeswohlgefährdungen, Aussetzung von Schuleingangsuntersuchungen usw.

Bei den Eltern sind es - je nach Situation - die Doppelbelastung durch Job und Home-Schooling der Kinder oder Home-Schooling zusammen mit Existenzängsten ${ }^{1}$ sowie Vereinsamung, zu wenige Unterstützungsangebote für Familien, mangelnde Bewegung und Zeit für sich, und speziell für Frauen die Genderfalle. Allen gemein ist jedoch die dauernde Überforderung, die über diesen langen zweiten Lockdown von erneut ca. sechs Monaten gravierende Spuren hinterlassen hat, aber auch eine gesellschaftliche und politische Schieflage - wenn nicht gar Spaltung - ans Tageslicht gebracht hat (Felber et al. 2021).

$1 \mathrm{Zu}$ den aktuellen Feststellungen siehe: Kluge, Regionaldirektor Europa der WHO (EURO-WHO 2021).

Ә Open Access. (๑) 2022 Nele Flüchter, Nicole Reese (c) BY-NC-ND Dieses Werk ist lizenziert unter der Creative Commons Attribution-NonCommercial-NoDerivatives 4.0 Lizenz. 


\section{Die wichtigsten Problemfelder auf Seiten der Kinder}

Im weiteren Verlauf werden die gravierendsten Probleme auf Seiten der Kinder dargestellt, wobei die psychischen und physischen Folgen aufgrund der verschiedenen Maßnahmen zusammengefasst erläutert werden. Neben der reinen Darstellung der Folgen liegt ein Hauptaugenmerk auf der mit den Maßnahmen einhergehenden Missachtung der Kinderrechte.

\subsection{Kinder als Virenschleudern}

Obwohl durch zahlreiche Studien widerlegt, hält sich in Deutschland das Narrativ der Kinder als Virenschleudern in der Corona-Pandemie. Immer wieder wiesen sowohl Politikerinnen und Politiker, als auch die Presse auf die Gefahr hin, dass Kinder Oma oder Opa anstecken können, da sie keinen Abstand halten oder als stille Überträger das Virus verbreiten. Durch diese Beschreibung in fast allen Medien wurden und werden immer noch Ängste in der Gesellschaft vor Kindern geschürt, was in vielen Fällen zu Ausgrenzung und Diskriminierung von Kindern führt. Kinder durften in den ersten Monaten der Pandemie beispielsweise häufig nicht einmal mit in Baumärkte oder Supermärkte, was zu einer Verschärfung der sowieso schon angespannten Betreuungssituation in den Familien geführt hat.

Warum hält sich dieses Narrativ so beständig in Deutschland und wie ist es entstanden? Der Erstimpuls lässt sich auf ein Strategiepapier des Bundesinnenministeriums vom 18.03.2021 zurückführen (vgl. Lehmann 2020). In diesem heißt es u. a.:

... Wenn sie dann ihre Eltern anstecken, und einer davon qualvoll zu Hause stirbt und sie das Gefühl haben, schuld daran zu sein, weil sie z. B. vergessen haben, sich nach dem Spielen die Hände zu waschen, ist es das Schrecklichste, was ein Kind je erleben kann. (BMI, Szenarienpapier, 2020)

Diese Kommunikation sollte eine Schockwirkung in der Gesellschaft erzielen und dafür sorgen, dass sich vor allem auch Kinder an die Corona-Regeln halten, um die ältere Generation zu schützen. Anstatt auf eine differenzierte Risikokommunikation zu setzen, also quasi den Goldstandard, der es der Bevölkerung einerseits erlaubt, informierte Entscheidungen zu treffen und andererseits schützendes bzw. lebenserhaltendes Verhalten zu fördern (Loss et al. 2021, 294), hat die Bundesregierung auf die gezielte Schockwirkung gesetzt. Diese bedient sie seitdem immer weiter, anstatt transparent zu machen, dass diese Einschätzung mit fortschrei- 
tendem Wissen nicht mehr aufrechterhalten werden kann. Insbesondere die aktuellen Debatten um die Impfpflicht von Kindern (stellvertretend für viele Beiträge siehe z. B. Ärzteblatt 2021) sowie ein erneutes Vorziehen der Weihnachtsferien zeigen, dass die Risikostrategie nicht angepasst und die Erkenntnisse der Wissenschaft immer noch nicht adäquat kommuniziert werden, sondern sogar in Frage gestellt bzw. schlichtweg missachtet werden und weiterhin Alarmismus betrieben wird, der Ängste und Beunruhigung in weiten Bevölkerungsteilen verstärkt (ebd.).

Diese Kommunikation hat bei vielen Kindern und Eltern Spuren in der Psyche hinterlassen. Familien haben Angst davor, sich mit den Großeltern zu treffen, zudem ist vielfach ein übersteigertes Hygienebewusstsein zu beobachten. In einem Interview sagte der Bielefelder Kinder- und Jugendpsychologe und -psychotherapeut Tobias Hecker:

Diese Stigmatisierung macht auch etwas mit der Psyche von Kindern, Jugendlichen und Familien: Es erhöht den Stress, vermittelt Ängste und Schuldgefühle, kurzum es zerstört die psychische Gesundheit. Das muss unbedingt aufhören. (Reese 2021)

\subsection{Bildungsverluste}

Nach Art. 28 Abs. 1 der UN-Kinderrechtskonvention (UN-KRK) erkennen die Vertragsstaaten das Recht des Kindes auf Bildung an. Die UN-KRK ist seit dem 18. Februar 1992 verbindlich geltendes Bundesrecht in Deutschland (BGBl II, 121). Um die Verwirklichung dieses Rechts auf der Grundlage der Chancengleichheit fortschreitend zu erreichen, haben die Vertragsstaaten u. a. die Entwicklung verschiedener Formen der weiterführenden Schulen allgemeinbildender und berufsbildender Art zu fördern, sie allen Kindern verfügbar und zugänglich zu machen und geeignete Maßnahmen wie die Einführung der Unentgeltlichkeit und die Bereitstellung finanzieller Unterstützung bei Bedürftigkeit zu treffen. ${ }^{2} \mathrm{Ob}$ ein Recht auf (schulische) Bildung auch aus dem Grundgesetz folgt, ist umstritten; das Bundesverfassungsgericht hat diese Frage bisher offengelassen. ${ }^{3}$ Allerdings enthalten die meisten Landesverfassungen ohnehin bereits einen solchen Anspruch. Art. 8 Abs. 1 S. 1 der Landesverfassung NRW bspw. erkennt ein Recht des Kindes auf Bildung ausdrücklich an.

Gleichwohl haben die Länder, angeschoben durch die Bundespolitik und infolge der o. a. Kommunikationsstrategie - entgegen anderslautender Zusagen im

2 SG München, Beschl. v. 17.08.2020 - S 8 AS 1135/20 ER, BeckRS 2020, 42222, Rn. 48.

3 BVerfG, Besch v. 27.11.2017 - 1 BvR 1555/14 -, juris, Rn. 25. 
Sommer 2020 - seit Ende des Jahres 2020 bzw. Anfang 2021 die Schulen flächendeckend geschlossen. Die Grundschulen wurden in Nordrhein-Westfalen erst am 22. Februar 2021 für den Wechselunterricht geöffnet, der bis zu den Osterferien lief. Nach den Osterferien wurden die Schülerinnen und Schüler in NordrheinWestfalen abermals eine Woche in den Distanzunterricht geschickt. Durch die Einführung der Bundesnotbremse konnten manche Kreise und Städte im Wechselmodell weitermachen, andere blieben bis Ende Mai im Distanzunterricht, bevor dann zum 31. Mai 2021 die Schulen in NRW in Vollpräsenz geöffnet wurden. Andere Bundesländer, allen voran Berlin, weigerten sich, die Schulen zu öffnen. Bildungssenatorin Scheeres begründete die Entscheidung, den Wechselunterricht fortzusetzen, damit, dass „die Ferien im Gegensatz zu manch anderen Ländern schon bald beginnen und sich der Aufwand einer Umstellung und Nutzen somit kaum lohnten“ (rbb24 2021).

Und damit nicht genug: Auch aktuell gibt es nicht wenige Politikerinnen und Politiker sowie Lehrer- und Elternverbände, die Wechselunterrichtsmodelle für den Herbst und Winter in Aussicht stellen oder fordern, obwohl es nach wie vor keine positive Evidenz dieser Maßnahme gibt (Young et al. 2021, 2).

Die Folgen der Schulschließungen sind immens. Im Rahmen des ifo-Bildungsbarometers 2020 kam heraus, dass sich Kinder im ersten Lockdown statt 7,4 Stunden täglich nur 3,6 Stunden mit schulischen Aktivitäten beschäftigt haben. Zugleich nahmen passive Tätigkeiten, wie Fernsehen schauen, Computer spielen und Handy nutzen zu (Wößmann et al. 2020, S. 28 f.). Interessanterweise gab es hier keine signifikanten Unterschiede zwischen Akademiker- und Nicht-Akademiker-Haushalten. Allerdings wird in dieser Studie deutlich, dass gerade bildungsschwache Schülerinnen und Schüler ihre schulischen Aktivitäten um eine halbe Stunde mehr reduzierten als leistungsstarke Kinder (ebd. S. 30). Über die Hälfte der Schülerinnen und Schüler (57\%) hatten in diesem Zeitraum seltener als einmal pro Woche gemeinsamen Unterricht für die ganze Klasse und sogar $67 \%$ der Kinder hatten weniger als einmal die Woche individuellen Kontakt mit einer Lehrkraft (ebd. S. 32f.).

Wößmann sagt hierzu: „Für einen nennenswerten Teil der Schülerinnen und Schüler fällt das Lernen während der Schulschließungen jedenfalls offensichtlich nahezu ganz aus" (ebd. S. 39). Da man in Deutschland davon ausgeht, dass jedes Jahr Bildung für 9 bis $10 \%$ des Lebenserwerbseinkommens von Bedeutung ist, führt dies zu der Annahme, dass die 3-monatigen Schulschließungen im Frühjahr 2020 das Erwerbseinkommen um ca. $3 \%$ negativ beeinflusst haben (ebd. f.). Die weiteren Schulschließungen für knapp ein halbes Jahr in 2021 führen demzufolge voraussichtlich zu weiteren Verlusten von ca. 5 bis $6 \%$ des individuellen lebenslangen Erwerbseinkommens. 
Neben dem individuellen Verlust an Einkommen führen die Einbußen an Lernkompetenzen aber auch zu einer Absenkung des volkswirtschaftlichen Einkommens insgesamt. $\mathrm{Zu}$ diesen langfristigen Folgekosten ausbleibenden Lernens kommen die umfangreichen zukünftigen Belastungen durch die massive Staatsverschuldung durch staatliche Hilfsmaßnahmen hinzu (ebd. S. 42).

Dass unter den Bildungsfolgen vor allem Kinder und Jugendliche aus sozial schwachen Elternhäusern leiden, hat $u$. a. eine Bildungsstudie der Universität Frankfurt belegt. Das Fazit der Forscherinnen und Forscher lautet:

Die durchschnittliche Kompetenzentwicklung während der Schulschließungen im Frühjahr 2020 ist als Stagnation mit Tendenz zu Kompetenzeinbußen zu bezeichnen und liegt damit im Bereich der Effekte von Sommerferien (Hammerstein et al. 2021, 2; Anger 2020; Gies-Thöne 2020).

Auch der Pisa-Studienleiter Schleicher formulierte die Folgen ähnlich drastisch:

Den Schaden für Gesundheit, Entwicklungs- und Berufschancen für Kinder, die dauerhaft nur eingeschränkten Kontakt zu Bildung und zu Freunden haben, den macht niemand wieder gut. Für Kinder aus bildungsfernen Schichten gibt es im Leben nur eine wirkliche Chance. Und das ist eine gute Schulbildung. (Schmid 2021)

Gleichwohl hat die Politik, obwohl bereits im Sommer und Herbst 2020 zahlreiche Studien die dramatischen Folgen für Kinder und Jugendliche im Hinblick auf Bildung erkannt und prognostiziert hatten, die Schulen seit Dezember 2020 bis teilweise in den Juni 2021 hinein geschlossen gehalten oder nur teilweise geöffnet, während Betriebe, Unternehmen und Verwaltungen weiter - ohne Home-OfficePflicht - geöffnet blieben.

Aber nicht nur die Politik hat sehenden Auges die Folgen in Kauf genommen, sondern auch die Gerichte. Diese verhalfen dem Recht auf Bildung im Wesentlichen nicht zum Durchbruch. ${ }^{4}$ Mehrheitlich zogen sich die Gerichte vielmehr darauf zurück, dass der Gesetz- bzw. Verordnungsgeber über einen sehr weiten Ermessens- und Prognosespielraum verfüge und dieser mehrheitlich beachtet worden sei bzw. zumindest in den anhängigen Eilverfahren der Überprüfung standhalte. Das OVG NRW stellte bspw. fest, dass Schulschließungen unabhängig vom Beitrag der Kinder an der Verbreitung des Virus und unabhängig von der Frage, ob die Schulschließungen überhaupt einen relevanten Beitrag

4 Siehe hierzu OVG NRW, Beschl. v. 22. Januar 2021 - 13 B 53/21.NE -, juris; Hamburgisches OVG, Beschl.v. 07. Mai 2021 - 1 Bs 73/21 -, juris; OVG Berlin-Brandenburg, Beschl.v. 19. April 2021 - OVG 3 S 26/21 -, juris; Hessischer VwGH, Beschl. v. 19. März 2021 - 8 B 309/21.N -, juris; Bayerischer VwGH, Beschl. v. 22. März 2021 - 20 NE 21.754 -, juris; OVG für das Land Schleswig-Holstein, Beschl. v. 11. März 2021 - 3 MR 14/21 -, juris. 
leisten können, gerechtfertigt seien, solange der Gesetzgeber nicht feststehende Tatsachen ignoriert. ${ }^{5}$ Besonders erschreckend an dieser Entscheidung ist jedoch, dass das Gericht ganz offen ausspricht, dass anhand der Relevanz für das öffentliche Leben Differenzierungen möglich seien und das Gericht und auch die Politik damit letztlich andere Lebensbereiche als relevanter einschätzen als die Bildung von Kindern (ebd.). Lediglich der Landesverfassungsgerichtshof NRW ${ }^{6}$ und das VG Berlin ${ }^{7}$ haben in zwei Entscheidungen leise Hoffnungen gemacht:

Laut Landesverfassungsgerichtshof NRW müssen zukünftige Abwägungsentscheidungen „erkennbar und plausibel vom Prinzip der größtmöglichen Schonung der Grundrechte der von den Freiheits- und Teilhabeeinschränkungen Betroffenen geleitet sein“. Zudem hat das Gericht anerkannt, dass mit der

Untersagung von Präsenzunterricht [...] hohe Belastungen im sensiblen Bereich der schulischen Bildung und der kindlichen und jugendlichen Entwicklung einhergehen, die - namentlich für jüngere Schülerinnen und Schüler - auch durch Distanzunterricht allenfalls unvollständig und nicht ohne soziale Verwerfungen kompensiert werden können. ${ }^{8}$

Geändert hat sich durch diese Entscheidung in der Praxis indes faktisch nichts.

\subsection{Schulbesuch unter höchstmöglichen Sicherheitsanforderungen}

Es ist wohl Konsens, dass Präsenzunterricht die beste Form des Lernens für die Mehrheit von Kindern und Jugendlichen ist. Auch Art. 28 der UN-KRK sagt, dass die unterzeichnenden Staaten das Recht des Kindes auf Bildung anerkennen. Die Vertragsstaaten verpflichten sich daher, alle geeigneten Maßnahmen zu treffen, um sicherzustellen, dass die Disziplin in der Schule in einer Weise gewahrt wird, die der Menschenwürde des Kindes entspricht und im Einklang mit diesem Übereinkommen steht.

Dies bedeutet, dass die Vertragsstaaten überdies alles zu unternehmen haben, um körperliche, aber auch geistige Gewaltanwendungen, wie Mobbing und verbale Aggression sowie öffentliche Demütigungen, die die genannten Rechte der Kinder verletzen, zu verhindern. Hiervon erfasst werden Handlungen durch die Institutionen und deren Personal selbst, aber auch Übergriffe oder Belästigungen durch andere Kinder. Es müssen also legislative Maßnahmen präventiver und

5 OVG NRW, Beschl. v. 22. Januar 2021 - 13 B 53/21.NE -, juris.

6 LVerfGH NRW, Beschl. v. 29. Januar 2021, Az. VerfGH 19/21.VB-1, www.nrwe.de.

7 VG Berlin, Beschluss vom 10. März 2021 - 3 L 57/21 -, juris.

8 LVerfGH NRW, Beschl. v. 29. Januar 2021, Az. VerfGH 19/21.VB-1, www.nrwe.de. 
repressiver Art vorgehalten werden, die derartige Eingriffe verhindern (Schmahl 2017, Art. 29 UN-KRK, Rn. 17).

Hier stellt sich die Frage, ob aktuellen Hygiene-Maßnahmen noch im Einklang mit der Kinderrechtskonvention stehen. Wir meinen nein, denn wenn Kindern der Zugang zum Präsenzunterricht nur gewährt wird, wenn diese dauerhaft Masken tragen, regelmäßig in der Klasse getestet werden und - wenn es nach einigen Politikerinnen und Politikern geht - am besten auch noch vollständig geimpft sind, stellt sich die Frage, inwiefern dies mit der Würde der Kinder vereinbar ist, insbesondere wenn man berücksichtigt, dass all diese Maßnahmen Arbeitnehmerinnen und Arbeitnehmern erst im Spätherbst 2021 auferlegt wurden.

Unter diesem Aspekt ist dem Ansinnen einiger Gruppen, allen voran der Lehrerverbände, eine Absage zu erteilen, wenn sie einerseits quasi die absolute Sicherheit in den Bildungseinrichtungen fordern, andererseits aber konsequent eine Impfpflicht für Schulpersonal ablehnen. Dazu beschwören sie bereits jetzt Wechselunterrichtsszenarien für den Herbst herauf (Wiarda 2021).

Vor allem wird bei der Forderung nach dem maximalen Schutz durch AHA-L Regeln, wobei es möglichst FFP2 Masken sein sollen, sowie anlassloser Tests, Wechselmodell, Luftfilter, Plexiglasabtrennungen und natürlich der Impfung der Kinder schlichtweg missachtet, dass für Kinder eine COVID-19 Erkrankung in aller Regel unproblematisch verläuft (DGPI 2021) und Schulen keine Pandemietreiber sind. Diese Erkenntnis ist in der Fachwelt inzwischen unbestritten. Vor diesem Hintergrund forderten bereits im Mai 2020 fünf große medizinische Fachgesellschaften in einer gemeinsamen Stellungnahme, Schulen und Kitas wieder vollständig zu öffnen (Walger et al. 2020). Derartige Einrichtungen stellen nach Auffassung der vorgenannten Verbände „per se keine Hochrisikoumgebung dar“ (ebd.), weil die Infektionsrate und Schwere der SARS-CoV-2-Infektion bei Kindern gering sind und auch das Übertragungsrisiko durch Kinder gering zu sein scheint.

Dies entspricht den Ergebnissen, die in jüngerer Zeit durch die CODAG-Berichte der LMU (CODAG Bericht Nr. 12 2021, 10 und 16) bestätigt wurden. Daneben existieren unzählige Studien, die belegen, dass die Öffnung von Schulen und Kinderbetreuungseinrichtungen als Ort der Begegnung keinen wesentlichen Beitrag zum Infektionsgeschehen leistet. An dieser Tatsache hat sich nach neuesten Feststellungen der WHO Europe nichts durch die Verbreitung von Mutationen geändert - weshalb diese auch der Auffassung ist, dass Schulschließungen nicht das Mittel der Wahl zur Kontrolle der Pandemie sind: "Even with the wider spread of more infectious variants, there is no evidence that schools contribute in a major way to community transmission: school closures will not control the pandemic" (WHO 2021).

Aufgrund all dieser Erkenntnisse ist nicht zu bestreiten, dass Kinder und Jugendliche auch - überwiegend passiv - am Infektionsgeschehen teilnehmen, aber 
gerade nicht aktiv wesentlich zur Verbreitung von SARS-CoV-2 beitragen. Mitunter gibt es Anzeichen dafür, dass der Schulbesuch gar einen protektiven Effekt aufweist und vor schweren Verläufen einer COVID-19-Erkrankung schützen kann (Dugas et al. 2020, 315). Sie belegen aber vor allem, dass ungeachtet der Rolle von Kindern jedenfalls die bislang in Schulen getroffenen Maßnahmen einer Verbreitung des Virus wirksam verhindern konnten, sodass angesichts der Tatsache einer fortschreitenden Impfung der erwachsenen Bevölkerung, vor allem des Personals an Schulen, gerade keine weiteren Maßnahmen erforderlich sind und Maßnahmen daher zurückgefahren werden können und müssen (Heudorf/Gottschalk 2021).

\subsection{Weitere psychische und physische Folgen durch die Corona-Maßnahmen}

Die vielen psychischen und physischen Folgen haben ihre Ursache in den verschiedenen Maßnahmen, die zur Pandemiebekämpfung eingesetzt wurden und werden. Zu nennen sind hier die beschlossenen, aber auch die diskutierten Kontaktbeschränkungen (wie die 1-Freund-Regel) oder die Quarantänepflicht für Reiserückkehrer, die anders als in den meisten Ländern keine Ausnahmen für Kinder vorsah. Weiterhin zu nennen sind die bereits mehrfach angesprochene angststiftende Kommunikation seitens der Politik, die Schließung von Sportplätzen und -hallen, Spielplätzen und Schwimmbädern, Musikschulen und Freizeiteinrichtungen und natürlich die geschlossenen Bildungseinrichtungen. Neben den soeben genannten Folgen führen diese Maßnahmen auch zu gravierenden Rechtsbeeinträchtigungen zu Lasten der Kinder.

\subsubsection{Psychische Folgen}

Für Kinder und Jugendliche fällt seit über einem Jahr ein Großteil ihrer Lebensräume weg. Dies führt zu ganz erheblichen psychischen Folgen. Die Copsy-Studie hat gezeigt, dass fast jedes dritte Kind nach einem Jahr Pandemie psychische Auffälligkeiten zeigt. Vor der Pandemie war es nur jedes fünfte Kind. Vor allem Sorgen und Ängste, aber auch depressive Symptome und psychosomatische Beschwerden haben zugenommen (Ravens-Sieberer 2020).

Die JuCo-Studie hat offenbart, dass selbst Jugendliche, die gut sozial vernetzt sind, oftmals Einsamkeitsgefühle, Verunsicherung und Überforderung fühlen (Andresen et al. 2020, 16). 
Zudem haben die fehlenden Möglichkeiten zur sportlichen Betätigung gerade im zweiten Lockdown dazu geführt, dass Kinder und Jugendliche sich weniger bewegten. Der Wegfall von Sportangeboten und das lange Sitzen während des digitalen Unterrichts sind Stressfaktoren (Fuchs u. Wunsch, Interview 2021). Anstatt sich zu bewegen und Stress abzubauen, nahm die Nutzung von Medien, gerade bei den 12- bis 19-jährigen um ca. $75 \%$ zu. Viele Kinder, vor allem Jungen, weisen einen pathologischen und riskanten Medienkonsum, gerade im Bereich des Gaming auf (DAK Studie 2020, 66; Bühring 2020, 337).

Darüber hinaus fehlte der Kontakt zu Gleichaltrigen, was zu depressiven Episoden und gesteigerten Ängsten führte. Bei jugendlichen Mädchen führte das zu deutlich mehr Fällen von Essstörungen, bis hin zu selbstverletzenden Verhaltensweisen und Suizidgedanken (Berndt/Schroeder 2021).

Nach Auffassung des Neurobiologen Hüther besteht aber auch die Gefahr, dass die andauernde Einschränkung der kindlichen Lebenswelt bei Kindern zu einem überangepassten Verhalten führt. Die Kinder verlieren das Gefühl für ihre Bedürfnisse. Überdies lernen sie, dass Berührungen gefährlich sein können und dass sie z. B. Oma oder Opa anstecken können und daraufhin das Bedürfnis nach Nähe und Kontakt dauerhaft unterdrücken. Den Kindern wird ihre kindliche Unbekümmertheit genommen und eine altersgerechte Entwicklung erschwert (Seidel 2021).

Alles in allem sind die psychischen Folgen immens und werden sich langfristig auswirken. Sie resultieren aus den andauernden Pandemiemaßnahmen, welche einen ganz erheblichen Eingriff in die Rechte der Kinder auf psychische und physische Gesundheit darstellen. Art. 24 UN-KRK ist ein individuelles Menschenrecht, verleiht also jedem einzelnen Kind Rechte und beinhaltet neben einer Leistungsdimension auch die klassische Abwehrfunktion, sodass der Staat alles zu unterlassen hat, was zu Lasten der Gesundheit des Kindes geht; zudem muss er Angriffe Dritter abwehren (Schmahl, Art. 24 UN-KRK, Rn. 1 ff.). Dieser Verpflichtung - das muss man in aller Deutlichkeit sagen - kommt der Staat in der Pandemie nur unzureichend nach, um nicht zu sagen, dass er in die Gesundheit gerade von Kindern und Jugendlichen nachteilig eingreift, denn Gesundheit ist nicht nur die Abwesenheit von SARS-CoV-2-Erkrankungen, sondern umfassend unter Einschluss des psychosozialen Wohlbefindens - zu verstehen.

\subsubsection{Physische Folgen}

Neben den psychischen und emotionalen Folgen greifen die Maßnahmen auch nachhaltig in die physische Gesundheit ein. Der Sportwissenschaftler Froböse bringt es auf den Punkt: „Wir produzieren die Kranken der Zukunft“ (Becker 2021). 
Kinder und Jugendliche befinden sich derzeit in einer außergewöhnlichen Situation, bei der gerade Sport helfen könnte, Stress abzubauen, die individuelle Gesundheit der Kinder zu fördern und damit auch das Immunsystem zu stärken (Ärztezeitung 2015). Dass gleichwohl fast sechs Monate kein regelhafter Sportbetrieb möglich war, führt, neben der vielfach zu beobachtenden Gewichtszunahme, auch zu Diabetes, Herz-Kreislauf-Erkrankungen sowie zum Verlust von motorischen und kognitiven Fähigkeiten, die für die gesunde Entwicklung von Kindern elementar sind (Becker 2021). So ermittelte eine Studie der TU München, dass neun Prozent der Kinder der Befragten an Gewicht zugelegt haben. Das betrifft besonders Schulkinder im Alter von zehn bis zwölf Jahren (Baumeister 2021). Das heißt, der Lockdown hat mittelbar Auswirkungen und verursacht enorme Kollateralschäden. So ist beispielsweise Adipositas nicht nur Treiber der Pandemie, sondern kostet jährlich 80.000 bis 100.000 Menschenleben in Deutschland (ebd.). Vor diesem Hintergrund ist der erneute Sport-Lockdown für ungeimpfte Kinder in vielen Bundesländern ein nicht zu rechtfertigender Eingriff in die Kinderrechte.

Auch der Verlust der Schwimmfähigkeit bzw. die fehlenden Möglichkeiten, Schwimmen zu lernen, führen zu erhöhten Gefahren. Besonders vom Ertrinken betroffen sind Kinder und junge Menschen. 2020 ertranken 18 Vorschulkinder und 5 Grundschulkinder (1 Kind mehr als 2019), während in den anderen Altersgruppen die Anzahl der Ertrunkenen abnahm (DLRG 2021).

\subsubsection{Gewalt und Missbrauch}

Während der Lockdowns gab es kaum Möglichkeiten, Vernachlässigung, Kindeswohlgefährdungen, im schlimmsten Falle (sexuellen) Missbrauch von Kindern und Jugendlichen zu entdecken. KiTa und Schule sowie andere Betreuungsangebote fielen als Schutz- und Kontrollinstanzen weg. Dadurch blieben viele Fälle unentdeckt, was man sehr gut daran erkennen kann, dass nach den Lockdowns die Zahlen der erfassten Kindeswohlgefährdungen sprunghaft anstiegen.

Durch fehlende Betreuungsmöglichkeiten während des Lockdowns stieg der Druck in den Familien. Dieser Druck äußerte sich häufig in gesteigerter Gewalt gegen Kinder (ntv 2021). Im Rahmen einer Studie kam ebenfalls heraus, dass 6,5 \% der Befragten zugaben, ihre Kinder körperlich bestraft zu haben (Steinert/Ebert 2021).

Die Kriminalstatistik zeigt, dass 2020152 Kinder gewaltsam zu Tode gekommen sind und es einen Anstieg um 53\% bei der sog. Kinderpornografie gibt (BKA 2021). Die Jugendämter in Deutschland haben im Jahr 2020 bei fast 60600 Kindern und Jugendlichen eine Kindeswohlgefährdung festgestellt. Das waren rund 5000 Fälle oder 9\% mehr als 2019 (destatis 2021). 
Die logische Konsequenz daraus ist, dass Bildungseinrichtungen und auch Hilfsangebote für Kinder und Jugendliche so lange wie möglich offengehalten werden müssen. Sie sollten als Letztes schließen. Vorher müssen alle Mittel zu Eindämmung der Pandemie in der Erwachsenenwelt ergriffen werden, wie z. B. eine echte Home-Office-Pflicht für Beschäftigte, die es in vielen anderen europäischen Ländern gab. Es darf nie wieder passieren, dass Bildungseinrichtungen erst nach den Freizeiteinrichtungen für Erwachsene öffnen und vor diesen geschlossen werden.

\subsubsection{Rechtliche Folgen}

Die Maßnahmen der Bundes- und der Landesregierungen konterkarieren die derzeitige Diskussion, ob Kinderrechte ins Grundgesetz aufgenommen werden sollten. Wie oben bereits ausgeführt, gilt die UN-KRK als einfaches Bundesrecht, und Kinder sind als Menschen in besonderem Maße bereits durch die Grundrechte geschützt. Es ist also kein Mangel an Rechten vorhanden, sondern ein Mangel an Bereitschaft, diesen Rechten auch Wirkung zu verleihen. Zusammenfassend ist festzustellen, dass insbesondere Art. 3, 24, 28 und 31 der UN-KRK massiv beeinträchtigt werden, d. h. das Recht auf vorrangige Berücksichtigung der Kinder, das Recht auf psychische und physische Gesundheit, das Recht auf Bildung und das Recht der Kinder auf angemessene Teilhabe an Freizeit, kulturellem und künstlerischem Leben. Daneben werden seit nunmehr 20 Monaten die Würde der Kinder aus Art. 1 GG und das Recht auf körperliche Unversehrtheit aus Art. 2 GG von Politik, Gesellschaft und Justiz massiv missachtet; ein unhaltbarer Zustand für eine freiheitliche Demokratie.

\section{Die wichtigsten Probleme auf Seiten der Eltern}

Aus Platzgründen soll hier nur kurz darauf verwiesen werden, dass auch Eltern übermäßig belastet werden (vgl. Institut für Demoskopie Allensbach 2021).

Während die einen Existenzängste hatten und haben und möglicherweise ihren Job sogar verloren haben, stellte sich für andere die schier unlösbare Aufgabe, Home-Schooling der Kinder und den Job unter einen Hut zu bringen. Während Eltern vor den Schulschließungen im Durchschnitt eine halbe Stunde pro Tag gemeinsam mit ihrem Kind beim Lernen für die Schule verbracht haben, ist der Wert während der Distanzphasen auf gut eine Stunde angestiegen und damit das Engagement der Eltern (Wößmann et al. 2020, S. 31). Allerdings variiert dies, je nach 
Alter und Veranlagung des Kindes stark. Neben der Lernbegleitung fallen aber auch weitere Aufgaben, wie Versorgung, Einkauf etc. an, die ebenfalls Zeit in Anspruch nehmen.

Den Familien bleibt während der Corona-Pandemie mithin nichts Anderes übrig, als zu funktionieren. Obwohl sie nach wie vor multipel belastet werden, hat die Politik ihnen mehrfach die Betreuung und Beschulung der Kinder während ihrer regulären Arbeitszeit zugemutet und damit konstatiert, dass es Lehr- und Erziehungspersonal eigentlich gar nicht braucht, denn das können ja die Eltern erledigen. Niemand käme auf die Idee, dies in anderen Berufsbereichen umzusetzen. Diese zusätzlichen Aufgaben gingen aber weder einher mit zusätzlichen echten Urlaubsansprüchen noch mit sonstigen Vorteilen, sondern es gab allenfalls eine anteilige Billigkeitsentschädigung nach dem IfSchG oder dem SGB V, verabschiedet mit hohen bürokratischen Hürden und vielen staatlichen Schlupflöchern und mangelnder Akzeptanz durch viele Arbeitgeberinnen und Arbeitgeber.

Während Regierung und Kirchen dazu aufgerufen haben, älteren Menschen beim Einkauf zu helfen, gab und gibt es keinen Aufruf zur Hilfe für Familien. Stattdessen werden Beratungsstellen geschlossen oder sind überlaufen. Familien bzw. Eltern befinden sich seit über anderthalb Jahren fast durchgehend in einer Doppelbelastungs-Falle und haben kaum noch Privatleben, denn selbst in den Zeiten, in denen Bildungseinrichtungen geöffnet waren, gab es häufig reduzierte Betreuungszeiten oder Quarantänen, die aufgefangen werden mussten. Kleine Hilfen in Form von Gutscheinen für Reinigungskräfte, Einkaufshilfen, Babysitterinnen und Babysitter oder Nachhilfekräfte wären sinnvoller als Einmalbeträge, die bei vielen Eltern wieder von der Steuer aufgefressen wurden.

„Die Menschen, vor allem die Familien [Hinzufügung der Autorinnen] in der europäischen Region brechen buchstäblich unter der Belastung von COVID-19 und seinen Folgen zusammen“, erklärte WHO-Regionaldirektor Hans Kluge (EURO-WHO 2021).

Gerade Frauen tappen hierbei in die Genderfalle, denn in den Familien lässt sich vermehrt eine Rückkehr zu traditionellen Rollenbildern beobachten: Die zusätzliche Sorgearbeit übernimmt zumeist die Frau, sodass auch die Frauen viel häufiger ihre Arbeitszeit verringert haben und damit über noch weniger eigenes Einkommen verfügen als zuvor. Da auch vorher zumeist Frauen in Teilzeit gearbeitet haben, fällt das Kurzarbeitergeld geringer aus und gerade Alleinerziehenden droht die Armutsfalle (Pokorny 2021). Im ersten Lockdown haben Frauen in rund $16 \%$ und damit im Vorjahresvergleich in etwa doppelt so vielen Familien weit überwiegend die Kinderbetreuung übernommen. Wenn Mütter im Home-Office arbeiten, erledigen sie auch mehr Sorgearbeit, während dies bei Vätern nicht der Fall ist (Jessen et al. 2021). 
Frauen sind somit doppelte Verlierer: Einerseits übernehmen sie den Großteil der Sorgearbeit, andererseits erhalten sie weniger Erwerbseinkommen (Kohlrausch/Zucco 2020). Für die Gleichstellung ist das ein herber Rückschlag.

\section{Fazit}

Familien, vor allem aber Kinder und Jugendliche sind die Verlierer der Pandemie in Deutschland. Zugleich hat der Umgang mit Familien und ihren Kindern gezeigt, dass Deutschland ein kinderfeindliches Land ist und Familien auch gesamtgesellschaftlich viel zu wenig Akzeptanz und Unterstützung erfahren. In der ganzen Pandemie ging es nie um die Kinder. Auch jetzt, wenn Politikerinnen und Politiker fordern, dass die Kinder doch jetzt mal dran sind und geimpft werden müssen, damit sie die Schule besuchen können, sind all diese Äußerungen letztlich ein Hohn und sollen nur eine weitere Rechtfertigung für weitere Eingriffe liefern. Claudia Bogedan, die ausgeschiedene Bildungssenatorin aus Bremen hat es in einem Spiegel-Interview zutreffend formuliert:

Wer monatelang eine Zero-Covid-Strategie fährt und immer noch härtere Maßnahmen fordert, kann doch jetzt nicht den moralischen Zeigefinger heben und uns erklären, wie sehr Kinder und Jugendliche unter den Schulschließungen gelitten haben. Da werde ich so sauer, das kann ich körperlich kaum aushalten. (Olbrisch 2021)

\section{Literatur}

Allensbach (2021) Ein Jahr Corona-Pandemie - Wie geht es Familien in der Krise? Zusammenfassung von Kernergebnissen mit Schaubildern zu einer repräsentativen Elternbefragung im Februar 2021, https://www.ifd-allensbach.de/fileadmin/IfD/sonstige_pdfs/8262_ Eltern_Corona_Krise_2021_fin.pdf. Zugriff am 30. September 2021.

Andresen S, Lips A, Möller R, Rusack T, Schröer W, Thomas S, et al (2020) Erfahrungen und Perspektiven von jungen Menschen während der Corona-Maßnahmen, Erste Ergebnisse der bundesweiten Studie JuCo, Universitätsverlag Hildesheim, Hildesheim, https://dx.doi. org/10.18442/120.

Anger C, Plünnecke A (2021) Schulschließungen - Auswirkungen und Handlungsempfehlungen, IW Report 44/2021, https://www.iwkoeln.de/studien/christina-anger-axel-pluenneckeschulschliessungen-auswirkungen-und-handlungsempfehlungen-513768.html. Zugriff am 30. September 2021.

Ärzteblatt (2021, 16. Juli) Debatte zum Coronaimpfen von Kindern: Ständige Impfkommission wehrt sich, Ärzteblatt online, https://www.aerzteblatt.de/nachrichten/125660/Debattezum-Coronaimpfen-von-Kindern-Staendige-Impfkommission-wehrt-sich. Zugriff am 19. August 2021. 
ÄrzteZeitung (2015, 08. Dezember) Immunsystem lässt sich durch Sport ankurbeln, https:// www.aerztezeitung.de/Medizin/Immunsystem-laesst-sich-durch-Sport-ankurbeln234783.html. Zugriff am 19. August 2021.

Baumeister K (2021, 02. Juni) „Corona befeuert eine andere Pandemie“, https://idw-online.de/ de/news769982. Zugriff am 19. August 2021.

Becker L (2021, 21. Januar) Ingo Froböse: „Wir produzieren die Kranken der Zukunft“ ipso.com, https://www.ispo.com/people/ingo-froboese-wir-produzieren-die-kranken-der-zukunft. Zugriff am 19. August 2021.

Berndt D, Schroeder V (2021, 19. Mai) Corona-Folgen: Wenn Verbote auf die Seele schlagen, SZ online, https://www.sueddeutsche.de/wissen/corona-lockdown-psyche-kinderjugendliche-krankheiten-1.5297873. Zugriff am 19. August 2021.

BKA (2021, 26 Mai) Vorstellung der Zahlen kindlicher Gewaltopfer - Auswertung der Polizeilichen Kriminalstatistik (PKS) 2020, https://www.bka.de/DE/Presse/Listenseite_ Pressemitteilungen/2021/Presse2021/210526_pmkindgewaltopfer.html. Zugriff am 19. August 2021.

BMI (2020) Szenarienpapier „Wie wir COVID-19 unter Kontrolle bekommen, https://www. bmi.bund.de/SharedDocs/downloads/DE/veroeffentlichungen/2020/corona/ szenarienpapier-covid19.pdf?_blob=publicationFile \&v=6. Zugriff am 30. September 2021.

Bühring P (2020) Onlinespiele und soziale Medien - Corona verstärkt die Sucht, Editorial Deutsches Ärzteblatt: 337, https://www.aerzteblatt.de/pdf.asp?id=214932. Zugriff am 30. September 2021.

CODAG (2021) Bericht Nr. 12 vom 01. April 2021, https://www.covid19.statistik.uni-muenchen. de/pdfs/codag_bericht_12.pdf.Zugriff am 30. September 2021.

DAK-Studie (2020) Mediensucht 2020 - Gaming und Social Media in Zeiten von Corona, DAKLängsschnittstudie: Befragung von Kindern, Jugendlichen (12-17 Jahre) und deren Eltern, https://www.dak.de/dak/gesundheit/dak-studie-gaming-social-media-und-corona2295548.html\#/. Zugriff am 1. Oktober 2021.

Destatis (2021, 21. Juli) 9 \% mehr Fälle: Jugendämter melden 2020 Höchststand an Kindeswohlgefährdungen, https://www.destatis.de/DE/Presse/Pressemitteilungen/2021/07/PD21_ 350_225.html. Zugriff am 30. September 2021.

Deutschen Gesellschaft für Pädiatrische Infektiologie (DPGI) (2021, 21. April) Stellungnahme von DGPI und DGKH zu Hospitalisierung und Sterblichkeit von COVID-19 bei Kindern in Deutschland, https://dgpi.de/wp-content/uploads/2021/04/Mortalitaet-Kinder-21_04_ 2021_korr.pdf. Zugriff am 30. September 2021.

DLRG (2021), Todesfälle durch Ertrinken 2020. Pressekonferenz des DLRG 09.03.2021, https:// www.dlrg.de/fileadmin/user_upload/DLRG.de/Fuer-Mitglieder/AA_DLRG2019/die_ dlrg/Presse/Statistik_Ertrinken/2020/dlrg-presse-ppt-ertrinken-2020.pdf. Zugriff am 19. August 2021.

Dugas M, Schrempf I, Ochs K, Frömmel C, Greulich L, Neuhaus P, et al (2020) Association of contact to small children with a mild course of COVID-19, International Journal of Infectious Diseases 100: 314-315, https://doi.org/10.1016/j.ijid.2020.09.003.

EURO-WHO (2021, 22. Juli) Psychische Gesundheit muss für alle ein Menschenrecht sein, WHO Veranstaltung, Pressemitteilung. Internetauftritt der WHO, Regionalbüro für Europa, https://www.euro.who.int/de/media-centre/sections/press-releases/2021/mentalhealth-should-be-a-human-right-for-all. Zugriff am 30. September 2021. 
Felber C, Fittkau B, Frommel M, Guérot U, Hamed J, Hanke M, et al (2021) COVID-19 ins Verhältnis setzen, Alternativen zu Lockdown und Laufenlassen, https://coronaaussoehnung.org/ wp-content/uploads/2021/07/Corona_ins_Verhaeltnis_setzen_Update_15-Juli-2021.pdf. Zugriff am 30. September 2021.

Fuchs S, Wunsch K (Interviewte) (2021) Bewegung macht Kinder und Jugendliche unempfindlicher gegen Corona-Frust - Ergebnisse der Mobilitätsstudie (MoMo) am Karlsruher Institut für Technologie - Campus-Report am 11.05.2021, https://publikationen.bibliothek.kit. edu/1000132653. Zugriff am 30. September 2021.

Geis-Thöne W (2020) Häusliches Umfeld während der Krise: Ein Teil der Kinder braucht mehr Unterstützung, IW-Report 15/2020, https://www.iwkoeln.de/studien/wido-geis-thoeneein-teil-der-kinder-braucht-mehr-unterstuetzung.html. Zugriff am 30. September 2021.

Hammerstein S, König C, Dreisörner T, Frey A (2021) Effects of COVID-19-Related School Closures on Student Achievement-A Systematic Review, https://www.frontiersin.org/articles/ 10.3389/fpsyg.2021.746289/full. Zugriff am 30. September 2021.

Heudorf U, Gottschalk R (2021) Zweiter Corona-Sommer - und dann?, Hessisches Ärzteblatt, 07-08, https://www.laekh.de/fileadmin/user_upload/Heftarchiv/Einzelartikel/2021/07_ 08_2021/Corona-Sommer.pdf. Zugriff am 30. September 2021.

Jessen J, Spieß CK, Wrohlich K (2021) Sorgearbeit während der Corona-Pandemie: Mütter übernehmen größeren Anteil - vor allem bei schon zuvor ungleicher Aufteilung, DIW Wochenbericht 9: 131-139, https://doi.org/10.18723/diw_wb:2021-9-1.

Kohlrausch B, Zucco Al (2020) Die Corona-Krise trifft Frauen doppelt, Weniger Erwerbseinkommen und mehr Sorgearbeit, Policy Brief WSI, Nr. 41.

Lehmann JF (2020) Bilder und Angst - über Politik, Wissenschaft und Kommunikation in Zeiten von Corona, https://www.uni-bonn.de/de/neues/bilder-und-angst-2013-ueber-politikwissenschaft-und-kommunikation-in-zeiten-von-corona. Zugriff am 30. September 2021.

Loss J, Boklage E, Jordan S, Jenny MA, Weishaar H, El Bcheraoui C et al (2021) Risikokommunikation bei der Eindämmung der COVID-19-Pandemie: Herausforderungen und Erfolg versprechende Ansätze, Bundesgesundheitsblatt - Gesundheitsforschung - Gesundheitsschutz: 294-303.

Ntv (2021, 21. Juli) Kindeswohl in Pandemie besonders gefährdet, ntv.de, mdi/ AFP, 2021, https://www.n-tv.de/panorama/Kindeswohl-in-Pandemie-besonders-gefaehrdetarticle22696292.html. Zugriff am 11. September 2021.

Olbrisch M (2021) Bremer Schulsenatorin über Bildungspolitik in der Pandemie: »lch habe so viel Wut im Bauch«, DER SPIEGEL 27/2021 / SPON, https://www.spiegel.de/politik/ deutschland/bremen-schulsenatorin-ueber-bildungspolitik-ich-habe-noch-so-vielwut-im-bauch-a-dec40816-0002-0001-0000-000178206292?context=issue. Zugriff am 19. August 2021.

Pokorny S (2021) Haushalt ist Frauensache? Familienleben vor und während der Corona-Pandemie, Berlin, https://www.kas.de/documents/252038/11055681/ Haushalt+ist+Frauensache+\%E2\%80\%93+Familienleben+vor+und+w\%C3 \%A4hrend+der+Corona-Pandemie.pdf/1cbfcaa6-7fed-7c35-526d-128ffec70981?t= 1626359751827. Zugriff am 30. September 2021.

Ravens-Sieberer U, Kaman A, Otto C, Adedeji A, Napp A, Becker M, et al (2021) Seelische Gesundheit und psychische Belastungen von Kindern und Jugendlichen in der ersten Welle der COVID-19-Pandemie - Ergebnisse der COPSY-Studie, Bundesgesundheitsblatt - Gesundheitsforschung - Gesundheitsschutz, https://doi.org/10.1007/s00103-021-032913. 
Rbb24 (2021, 26. Mai), Berliner Politik streitet über Schulöffnung vor den Ferien, Inforadio, https://www.rbb24.de/politik/thema/corona/beitraege/2021/05/berlin-forderunggruene-gebel-liecke-neukoelln-schuloeffnung-.html. Zugriff am 19. August 2021.

Reese N (2021, 11. Mai) Interview mit Prof. Dr. Tobias Hecker: Auswirkungen der Corona-Maßnahmen bei Kindern und Jugendlichen. \#laut für Familien, https://lautfuerfamilien. de/interview-mit-prof-dr-tobias-hecker-auswirkungen-der-corona-massnahmen-beikindern-und-jugendlichen. Zugriff am 19. August 2021.

Schmahl S (2017) Kommentar zur UN-Kinderrechtskonvention, 2. Auflage, Nomos Verlag, München.

Schmid H (2021, 10. Februar) Folgen von Schulschließungen „Den Schaden macht niemand wieder gut“, Tagesschau.de, https://www.tagesschau.de/ausland/oecd-schulen-coronavirusschliessungen-101.html. Zugriff am 19. August 2021.

Seidel $\ddot{A}$ (2021, 24. Januar) Neurobiologe Hüther über Lockdown-Folgen „Schule ist der Ort, wo Kinder ihre tiefsten Bedürfnisse stillen“, Deutschlandfunk.de, https://www. deutschlandfunk.de/neurobiologe-huether-ueber-lockdown-folgen-schule-ist-der-100. html. Zugriff am 19. August 2021.

Steinert J, Ebert C (2021) Gewalt an Frauen und Kindern in Deutschland während COVID-19-bedingten Ausgangsbeschränkungen: Zusammenfassung der Ergebnisse, https://drive.google.com/file/d/19Wqpby9nwMNjdg04_FCqqlfYyLJmBn7y/view.Zugriff am 30. August 2021.

Walger P, Heininger U, Knuf M, Exner M, Popp W, Fischbach T, et al (2020) Kinder und Jugendliche in der COVID-19 Pandemie: Schulen und Kitas sollen wieder geöffnet werden. Der Schutz von Lehrern, Erziehern, Betreuern und Eltern und die allgemeinen Hygieneregeln stehen dem nicht entgegen (DHKH-Studie), https://dgpi.de/wp-content/uploads/ 2020/05/Covid-19_Kinder_Stellungnahme_DGKH_19_05_2020_final_v6.pdf, Zugriff am 30. September 2021.

WHO (2021, 09. März) Findings of Fourth meeting of the Technical Advisory Group on Safe Schooling during the COVID-19 Pandemic, Regionalbüro für Europa, https://apps.who. int/iris/bitstream/handle/10665/340359/WHO-EURO-2021-2197-41952-57642-eng.pdf? sequence=1\&isAllowed=y. Zugriff am 30. August 2021.

Wiarda JM (2021, 23. Juli) Die Macht eines Narrativs, Webblog, https://www.jmwiarda.de/2021/ 07/23/die-macht-eines-narrativ. Zugriff am 19. August 2021.

Wößmann L (2020) Folgekosten ausbleibenden Lernens: Was wir über die Corona-bedingten Schulschließungen aus der Forschung lernen können, ifo Schnelldienst: 38-44.

Wößmann L, Freundl V, Grewenig E, Lergetporer P, Werner K, Zierow L (2020) Bildung in der Coronakrise: Wie haben die Schulkinder die Zeit der Schulschließungen verbracht, und welche Bildungsmaßnahmen befürworten die Deutschen? ifo Schnelldienst: 25-39.

Young BC, Eyre DW, Kendrick S, White C, Smith S, Beveridge G (2021) Oxford-Studie: A cluster randomised trial of the impact of a policy of daily testing for contacts of COVID-19 cases on attendance and COVID-19 transmission in English secondary schools and colleges, https://www.medrxiv.org/content/10.1101/2021.07.23.21260992v1. Zugriff am 19. August 2021. 